\title{
El índice de cobertura de servicios como instrumento de gestión: su utilización en el proceso de construcción de cuadros de mando integrales
}

\author{
José Manuel Rivera OTERO \\ Universidad de Santiago de Compostela \\ josemanuel.rivera@usc.es \\ María PEReIRA LóPEZ \\ Universidad de Santiago de Compostela \\ mariapereira.lopez@gmail.com \\ Erika JARÁIZ GULÍAS \\ Universidad de Santiago de Compostela \\ erika.jaraiz@usc.es
}

Recibido: 09/04/2015

Aceptado: 15/05/2015

\begin{abstract}
Resumen
La importancia y necesidad de contar con mecanismos de evaluación de los servicios públicos ha sido una constante en el ámbito de la gestión pública en las últimas dos décadas. Deudores en muchos casos del ámbito privado, como consecuencia del predominio de algunos paradigmas teóricos, se han puesto en marcha con mayor o menor éxito diferentes propuestas.

Esta situación, si cabe más acuciante en el ámbito municipal y en un contexto de crisis económica donde los ciudadanos demandan de las instituciones un mayor nivel de transparencia y rendición de cuentas, no sólo de los recursos sino también de cómo llevan a cabo la gestión de los servicios públicos; nos ha invitado a diseñar una metodología mixta de evaluación de la cobertura de los servicios públicos municipales.

Una metodología que debe servir no sólo para medir y/o evaluar sino también para construir cuadros de mando en diferentes niveles, en los cuales el Índice de Cobertura de Servicios (ICS), se perfila como pieza fundamental para el análisis de los servicios, fusionando elementos de carácter estructural y elementos de carácter perceptivo.
\end{abstract}

Palabras clave: Índice de Cobertura de Servicios, umbrales de cobertura, sistema de indicadores, Cuadro de Mando Integral, evaluación de políticas públicas.

${ }^{1}$ Nuestro más sincero agradecimiento a la Diputación de A Coruña, la realización de esta investigación no habría sido posible sin su apoyo a través de diferentes proyectos. Agradecemos también a los gestores y técnicos de la misma, así como a los técnicos de los ayuntamientos que han estado implicados desde el primer momento en este proyecto. 


\title{
The Coverage Services Index (CSI) as management tool: the use in the construction process of Balanced Scorecard
}

\begin{abstract}
The importance and need for evaluation mechanisms of public services has been a constant in the field of public administration in the last two decades. Debtors in many cases the private sector, reflecting the predominance of some theoretical paradigms have been implemented with varying degrees of success, different proposals.

This situation, all the more pressing at the municipal level and in a context of economic crisis where citizens demand to institutions a greater level of transparency and accountability, not only resources but also how performs management public services; it has invited us to design a mixed assessment methodology coverage of municipal utilities.

A methodology to be used not only to measure and/or evaluate, but also to build balanced scorecards at different levels, in which the construction of Index of Services Coverage (ICS) is emerging as a cornerstone for the analysis of services, mixed structural elements and perceptive elements.
\end{abstract}

Key words: Index of Services Coverage, coverage thresholds, system of indicators, Balanced Scorecard, evaluation of public policies.

\section{Introducción}

En un contexto marcado por la crisis económica y por las consecuencias que la misma tiene no sólo en la vida de los ciudadanos sino también y sobre todo en la gestión de las políticas públicas que llevan a cabo los gobiernos, los primeros reclaman de estos últimos un mayor nivel de transparencia, respecto de los recursos destinados a tal fin, así como respecto a la forma en la que se lleva a cabo la gestión pública. Llegado este punto, la utilización de indicadores o herramientas metodológicas como los cuadros de mando, que faciliten la gestión y evaluación de las políticas y servicios que prestan las administraciones públicas, adquiere una relevancia crucial.

El uso y empleo de indicadores de gestión y evaluación en las entidades públicas ha empezado a tener una importante repercusión como medio de evaluación y rendición de cuentas de dichas entidades ante la sociedad, así como herramienta de improvement de la gestión que llevan a cabo las mismas; por lo que no resulta extraño que estos indicadores estén siendo utilizados por los gobiernos de los países más avanzados en materia de evaluación de las actuaciones y políticas públicas (Brusca Alijarde, 1997). En el caso español podemos hablar de dos etapas en la evolución de este tipo de estudios de carácter evaluativo, una primera etapa desde finales de los años ochenta a mediados de los noventa, de marcado carácter teórico, donde comienza a plantearse una obsesión por el logro de la eficacia y la calidad de los servicios (Colino y Del Pino, 2008); y una segunda etapa, desde mediados de los años noventa hasta la actualidad, caracterizada por un marcado carácter empírico².

${ }^{2}$ Autores como Font y Gomá identifican en este último período, cuyo comienzo sitúan en 1995, una tendencia hacia la innovación democrática, en la cual se tienden a importar mecanismos del sector privado (Font y Gomá, 1999). 
La evaluación en el sector público presenta importantes inconvenientes (Wolf, 1995) tales como la existencia de objetivos diversos, el gran número de actividades realizadas, el tamaño de las actuaciones públicas, la menor experiencia en la aplicación de medidas de eficacia y eficiencia, el temor a la evaluación; a los que se unen la amplitud y falta de concreción de los objetivos (condicionados por cuestiones de cariz político) o la dificultad de desagregación con el fin de realizar una operativización (De Pablos Escobar y Valiño Castro, 2000). A esta última se unen otras deficiencias respecto de la medición, señaladas por Sorensen y Grove (1977), tales como el intervalo de medida para realizar las mediciones, los múltiples resultados de un programa, los efectos entre distintos colectivos, las interrelaciones entre programas o los factores del entorno.

Los indicadores de gestión se perfilan como instrumentos claramente útiles para la evaluación del desempeño dentro del sector público y facilitan, por tanto, el proceso de transparencia y rendición de cuentas ante la ciudadanía. El trabajo que aquí se presenta, contextualizado dentro del paradigma del Nuevo Servicio Público (NSP), expone la importancia del diseño de un cuadro de mando integral de indicadores de ámbito municipal de los diferentes servicios públicos, así como la definición y construcción de índices sintéticos en el ámbito de la cobertura o prestación de dichos servicios: el Índice de Cobertura de Servicios (ICS).

\section{Sistemas de indicadores y cuadros de mando, herramientas de gestión y evaluación pública}

Como se ha puesto de manifiesto en líneas anteriores, la importancia del diseño y desarrollo de sistemas de indicadores y cuadros de mando se desvelan como mecanismos indispensables para la gestión y evaluación en el ámbito del sector público, permitiendo la identificación de elementos fuertes y débiles dentro de las diferentes áreas o servicios, y por tanto, la posibilidad de introducir mejoras o cambios que permitan optimizar los recursos destinados.

A nivel normativo, en España las principales cuestiones en torno a la importancia y desarrollo en la práctica de este tipo de sistemas de indicadores de gestión se encuentran recogidas en la Ley 18/2001 de 12 de diciembre General de Estabilidad Presupuestaria, en la Ley 47/2003 de 26 de noviembre General Presupuestaria, en la Ley 57/2003 de 16 de diciembre de medidas para la Modernización del Gobierno Local; así como en la Orden EHA/4041/2004 de 23 de noviembre, por la que se aprueba la Instrucción del modelo normal de contabilidad local y los reales decretos 951/2005, de 29 de julio, por el que se establece el marco general para la mejora de la calidad en la Administración General del Estado y 2/2007, de 28 de diciembre, por el que se aprueba el texto refundido de la Ley General de Estabilidad Presupuestaria.

En los dos primeros textos legales mencionados, se recogen como uno de los principios reguladores de la actividad presupuestaria, el principio de eficiencia en la asignación y utilización de los recursos públicos, que difícilmente puede aplicarse sin la ayuda de indicadores de gestión. Además, es importante destacar, que en la citada 
Orden EHA/4041/2004, se incluye como novedad, la exigencia a todos aquellos municipios de más de 50.000 habitantes y a las demás entidades locales de ámbito superior, la inclusión en el apartado veintiuno de la memoria, de indicadores de gestión que permitan evaluar la economía, eficacia y eficiencia en la prestación de al menos, los servicios financiados con tasas o precios públicos (Navarro Galera, Ortiz Rodríguez y López Hernández, 2006). Esta exigencia fomenta y facilita claramente la rendición de cuentas por parte de las entidades públicas ante la ciudadanía, uno de los principios rectores del paradigma del Nuevo Servicio Público.

Los indicadores representan instrumentos de gran utilidad para evaluar la mejora de la calidad en los servicios prestados por las administraciones públicas y en sus actuaciones, contribuyendo así, a la reforma del sector público tradicional marcada, fundamentalmente, por la introducción de la competencia y el mercado y por la gestión orientada hacia el ciudadano (López Hernández y Ortiz Rodríguez, 2004). Orientación que consideramos, debe ser prioritaria en la elaboración y/o construcción de sistemas de estas características, situando al ciudadano en el centro de la gestión y evaluación de las políticas que las administraciones llevan a cabo; como copartícipes directos de las mismas.

Son muchas las instituciones y organizaciones - GASB, Audit Commision, AECAque han abordado las funciones que el empleo de indicadores cumple en el sector servicios. Funciones que podrían sintetizarse de la siguiente forma: permiten la toma de decisiones políticas y técnicas, facilitan información sobre la gestión, mejoran el proceso de rendición de cuentas, apoyan el proceso presupuestario, y permiten la comparación entre unidades de análisis, así como la introducción de mejoras en aquellas organizaciones que los emplean. Su uso y análisis permite a las organizaciones de cara al futuro, ayudar a detectar aquellas funciones, programas, centros o servicios que deberían ser objeto de especial atención (Torres Pradas, 1991); hecho que se revela fundamental de cara a una redistribución y optimización de recursos y a una redefinición de objetivos.

A pesar de la complejidad del término indicador en sí mismo y del amplio número de definiciones y clasificaciones que podemos encontrar, el GASB (Governmental Accounting Standard Board) propuso en el año 1987 el siguiente listado de características que un indicador debe cumplir para ser adecuado y reflejar fielmente aquellos aspectos del servicio que pretende medir: comprensibilidad o claridad, fiabilidad o verificabilidad, relevancia, oportunidad, consistencia y comparabilidad (Governmental Accounting Standards Board, 1987). A estas cualidades podríamos añadir las de objetividad y accesibilidad, propuestas por la AECA (1999). Además, en cuanto al conjunto o catálogo de indicadores, la Audit Commission (2000) señala seis principios a tener en cuenta para su elaboración: claridad en la finalidad, enfoque, encuadre, equilibrio, mejora regular y fortalecimiento de los indicadores.

En la actualidad, son varias las entidades que demuestran interés por construir catálogos de indicadores recopilados o creados $a d$ hoc para la medición de los servicios o bien de los elementos internos de éstos, conformando lo que se ha venido en denominar Cuadro de Mando Integral (CMI) o Balanced Scorecard (Kaplan, 2000) (Kloot y 
Martin, 2000) (Kaplan y Norton, 2001a) (Kaplan y Norton, 2001b)3. El Cuadro de Mando Integral (CMI) puede ser definido como una «metodología o técnica de gestión, que ayuda a las organizaciones a transformar su estrategia en objetivos operativos medibles y relacionados entre sí, facilitando que los comportamientos de las personas clave de la organización y sus recursos se encuentren estratégicamente alineados» (Amo Baraybar, 2011). En cualquier caso, el CMI, tal y como lo entendieron Kaplan y Norton, va más allá de ser un sistema de indicadores tradicionales, sino que construye una metodología para implantar la estrategia a todos los niveles dentro de una organización (Kaplan y Norton, 1996), convirtiéndose en herramientas que facilitarán el proceso de toma de decisiones, haciendo hincapié en la vinculación de la medición a un mapa de estrategia (Kaplan y Norton, 2006a) (Kaplan y Norton, 2006b) (Kaplan, 2012).

Esta técnica o metodología surgida en el ámbito privado y ligada a la gestión estratégica de las empresas o compañías, espacio en el que se presentan la mayoría de aplicaciones prácticas de la misma hasta el momento; en las organizaciones que no poseen ánimo de lucro, como es el caso de los ayuntamientos, la implantación del CMI, si bien se ha llevado a cabo, no ha experimentado, de momento, la evolución mencionada en el sector privado. Aunque las entidades públicas locales, unidades de análisis objeto de nuestra investigación, no tienen como objetivo fomentar la riqueza, sí requieren de instrumentos flexibles que les permitan manejar sus recursos, en función de las necesidades de sus usuarios y con ello, lograr el propósito encomendado (Bastidas E. y Ripoll, V., 2003): ofrecer a los ciudadanos una adecuada prestación o cobertura de los servicios públicos. Esta metodología, por la que la literatura especializada ha mostrado gran interés desde los años 90 (Hoque, 2014), facilita a las entidades públicas mejorar su gestión concentrándose en conseguir un alto nivel de satisfacción para lograr, de esta forma, prestar un servicio más eficiente y efectivo (Brignall, S. y Modell, S., 2000); siempre y cuando esta aplicación, deudora en exceso de las peculiaridades del ámbito privado, sea adaptada al ámbito público y orientada al ciudadano. Este hecho también es señalado por Kloot y Martín (2000), al poner de relieve el hecho de que los gobiernos necesitan de mejores medios para evaluar su desempeño respecto a sus objetivos, los cuáles con frecuencia, no se establecen en términos financieros; siendo necesario el empleo de otro tipo de indicadores, que deberán poner en valor las necesidades y demandas expresadas por los ciudadanos, herederos por derecho, de los servicios públicos que reciben.

Este tipo de herramientas suelen estar compuestas por un amplio número de indicadores de diversa índole, entre ellos: indicadores de inputs, indicadores de outputs, indicadores de presupuesto, indicadores de los estados contables, indicadores de orga-

${ }^{3}$ Previo a la aparición de los primeros artículos de Kaplan y Norton donde proponían la metodología del Cuadro de Mando Integral, se empleaba el Cuadro de Mando, una aportación procedente de la doctrina francesa. El CMI supuso una innovación metodológica respecto al Cuadro de Mando al introducir una visión metodológica desde cuatro perspectivas de una organización: la perspectiva financiera, la perspectiva del cliente/usuario, la perspectiva de los procesos internos y la perspectiva del crecimiento y el aprendizaje. 
nización, indicadores sociales, indicadores de entorno o impacto e indicadores de economía, eficiencia y eficacia (Torres Pradas, 1991). Todos ellos importantes desde el punto de vista de la gestión, pues su empleo conjunto se revela fundamental para la obtención de un adecuado análisis de la situación de cada uno de los servicios públicos en las diferentes entidades analizadas.

Para que la aplicación de un sistema de información a través del empleo de indicadores en una entidad pública sea útil y contribuya al proceso de toma de decisiones, en lo que a políticas y servicios públicos se refiere, debe establecerse una dependencia respecto de una serie de factores entre los cuales se encuentran: la institucionalización, entendida como máximo grado de aceptación por parte del personal implicado en el mismo y por otro lado, la factibilidad de cálculo, identificada como la posibilidad de la referida entidad para generar, de modo continuo en el tiempo, los datos necesarios para la utilización continuada del sistema de indicadores establecido (Navarro Galera, Ortiz Rodríguez y López Hernández, 2006) ${ }^{4}$. Dada la importancia de la institucionalización y la estandarización, sería de gran utilidad el hecho de que los indicadores o catálogos de indicadores estuvieran sometidos a un proceso de control interno y externo como mecanismo de garantía de rendición de cuentas frente a los ciudadanos; además sería conveniente que su cálculo y desarrollo se recogiera en una norma legal que facilitara así su fiscalización y publicación (Batet Lamaña y Mora Puigv, 2001) (Batet i Lamaña, Pastos i Millan, Carreras i Puigdengol y Mora i Puigví, 2001) .

Por todo lo expuesto, a la hora de definir los indicadores o las baterías de indicadores, dada la insuficiencia de la información que facilitan los indicadores de carácter financiero y/o presupuestario para la evaluación de las actuaciones de las entidades públicas, es necesario tener en cuenta otro tipo de principios o criterios esenciales de la gestión pública, como eficacia, eficiencia, economía, equidad, excelencia, entorno y evolución sostenible ${ }^{6}$, que permitan la definición o elaboración de nuevos indicadores.

${ }^{4}$ La idea de aplicación continua en el tiempo o perdurabilidad se ha tenido muy presente a la hora de construir la metodología que vertebra nuestra investigación, pues entendemos que sólo la validez temporal de una metodología, permite su aplicación continuada y favorece la obtención de resultados fiables que garanticen la generalización y en última instancia, la comparabilidad entre unidades de análisis a lo largo de una serie temporal.

${ }^{5}$ Teniendo en cuenta estas afirmaciones, la concepción de esta investigación propone la creación de una base de datos que aglutine y sistematice los resultados que año a año se recojan en cada una de las unidades de análisis, sobre los diferentes indicadores que se han definido para cada uno de los servicios públicos analizados.

${ }^{6}$ Definimos estos términos de la siguiente forma:

- Economía: condiciones de acceso a los recursos financieros, humanos y materiales de un determinado organismo.

- Eficacia: grado de satisfacción de los objetivos fijados en los programas de actuación en relación a lo previsto.

- Eficiencia: relación existente entre los servicios prestados y los recursos empleados a tal efecto.

- Equidad: posibilidad de acceso a los servicios públicos de los grupos sociales menos favorecidos en comparación con las posibilidades medias del resto de la población.

- Excelencia: remite a la noción de calidad de los servicios, desde la óptica del usuario.

- Entorno: espacio y condicionantes del mismo en el que se desarrolla el servicio.

- Evolución sostenible o Sostenibilidad: capacidad de mantener el servicio con una calidad aceptable a lo largo del tiempo. 
Esto plantea ciertos problemas, pues si bien los controles financieros y de legalidad en las administraciones públicas - tienen nítidamente definida su finalidad, los controles de eficacia, eficiencia y economía, precisan de la configuración de sus propios criterios de actuación, los cuales, además de diferir notablemente de los empleados en los dos tipos de controles anteriores, no parecen encontrarse claramente definidos (López Hernández y Ortiz Rodríguez, 2004). En el ámbito anglosajón a los tres principales criterios, anteriormente mencionados, - economía, eficiencia y eficacia - se añaden los de equidad, excelencia, entrepreneurship (asunción de riesgos propios de una organización), expertise (habilidad en un campo concreto de conocimiento) y electability (elección cuidadosa de un grupo de trabajo) (López Hernández y Ortiz Rodríguez, 2004).

La obligación de responder a una mayor demanda ciudadana de información respecto a la gestión que de los recursos llevan a cabo las entidades públicas, es lo que ha generado la necesidad de establecer mecanismos útiles de presentación de la misma (Batet Lamaña y Mora Puigví, 2001) ${ }^{7}$. Para esta labor, los indicadores son una potente herramienta, pues como mencionamos, facilitan la comparabilidad objetiva, temporal y entre unidades de análisis, facilitando información de interés para los gestores públicos de cara a la definición de los objetivos de cualquier tipo. Respecto a este último aspecto, la comparabilidad, debemos de tener presente que ésta no debe tener por objetivo último el establecer ránquines entre las unidades de análisis - en este caso concreto las entidades municipales - sino aspirar únicamente a buscar y establecer las diferencias en la prestación o cobertura de los servicios públicos entre dichas entidades, para intentar solventarlas o reconducirlas. Además, en la medida en que seamos capaces de mantener en el tiempo dicha comparabilidad entre unidades de análisis, la utilidad de este instrumento de medida aumentará considerablemente, tendiendo a perdurar en el tiempo y a ofrecer resultados cada vez más consistentes y fiables.

Pero para garantizar la comparabilidad de un indicador, éste necesita de un referente que debe ser consecuente con su construcción misma. La labor de encontrar y determinar este tipo de referentes es ardua por diversas razones y para facilitarla, entran en juego las denominadas técnicas de benchmarking o técnicas de comparación de indicadores; procesos mediante los cuales se identifican e incorporan en el análisis, las mejores prácticas para mejorar el desempeño ${ }^{8}$ (Keehley, Medlin y MacBride, 1997).

A pesar de todas las ventajas y potencialidades descritas, también se han puesto de relieve las limitaciones y problemas que la aplicación de indicadores en el ámbito público acarrea, las cuales en cualquier caso, pueden ser paliadas con la introducción de ciertas mejoras en la definición y desarrollo de los mismos, contando para ello con

${ }^{7}$ Recordemos que el paradigma del Nuevo Servicio Público defiende la importancia que para la construcción de la ciudadanía tiene la información y consecuentemente, la transparencia institucional; como mecanismo para hacer parte de la gestión al ciudadano, un ciudadano que para ser agente activo necesita estar y ser informado.

${ }^{8}$ Entendiéndose por mejores prácticas, aquellos procesos que han tenido éxito a través del tiempo con resultados cuantificables, reconocidos y repetitivos, en los que se ha utilizado la innovación y cuyas técnicas pueden ser generalizables a distintas áreas y culturas organizacionales. 
la combinación de técnicas de análisis cuantitativo y cualitativo, así como la fusión de elementos procedentes de diferentes fuentes. El GASB señala como principales desventajas o limitaciones las siguientes: 1) la obtención de datos; 2) la realización de comparaciones; 3) la complejidad para la evaluación de situaciones con múltiples inputs y outputs; 4) la delimitación del conjunto y número de indicadores óptimo; 5) la imposibilidad de que un único indicador sea capaz de informar adecuadamente sobre los resultados de un servicio; 6) la incapacidad de servir, por sí solos -los indicadorespara conocer el porqué de la situación; 7) la dificultad de ofrecer información sobre procesos o estrategias; 8) la complejidad para determinar la idoneidad de un indicador para la medición de una actividad; 9) el impedimento a la hora de discernir entre resultados intermedios o finales; 10) la dificultad para que reflejan claramente, si los objetivos fijados son o no los más adecuados a las pretensiones y deseos de los ciudadanos; 11) el hecho de que en ocasiones la información que proporcionan resulte incompleta a los efectos de evaluar de forma global la responsabilidad política y 12) la mala interpretación de los indicadores, tomados en muchas ocasiones como fin y no como instrumentos de medida (Governmental Accounting Standards Board, 1994).

Finalmente, debemos de tener claro que no existen indicadores de uso universal, por lo que muchas de las cuestiones planteadas en líneas anteriores deben tomarse como principios guía que ayuden a elaborar catálogos y baterías de indicadores específicas para los diferentes servicios y unidades de análisis, puesto que las peculiaridades juegan en esta construcción un importante papel. Por ello, los catálogos de indicadores o Cuadros de Mando Integral, a pesar de las posibles limitaciones señaladas, deben entenderse no cómo un objetivo final sino como un potente instrumento de análisis y evaluación en sí mismos. Si bien esta herramienta, el CMI, debe seguir siendo desarrollada tanto teórica como empíricamente, se perfila como valiosa dentro del ámbito público pues permitirá seguir proporcionando a las organizaciones, una opción valiosa como mapa estratégico, facilitando la implementación de la política, y desvelándose como una herramienta de control y rendición de cuentas de la organización (Hoque, 2014).

\section{Diseño de una metodología mixta de evaluación de servicios}

El Equipo de Investigaciones Políticas de la Universidad de Santiago de Compostela ha venido desarrollando desde hace algunos años, un amplio proyecto de investigación para la evaluación de la prestación o cobertura que de los servicios públicos llevan a cabo los ayuntamientos. Un proyecto integral que ha permitido y permite, pues se siguen avanzando en la actualidad nuevas líneas de trabajo, la construcción de una metodología robusta de análisis y evaluación de los servicios públicos municipales, a través de dos elementos: el diseño y aplicación del denominado Índice de Cobertura de Servicios y la elaboración de un Cuadro de Mando Integral de los diferentes servicios.

El ICS es un índice sintético que proporciona el grado de cobertura de los servicios públicos que prestan los municipios, poniendo en relación los aspectos de carácter objetivo o indicadores de diagnóstico, elemento estructural; con la priorización que los ciudadanos hacen de la incidencia de los componentes internos que definen a estos ser- 
vicios, respecto de los niveles de satisfacción con dichos servicios expresados por ellos mismos, elemento perceptivo. No se trata por tanto de un índice de satisfacción al uso ${ }^{9}$, sino un índice que pone en conexión dos fuentes de información en principio dispares, pero claramente complementarias, que permiten obtener una visión precisa de la situación en la que se encuentra cada uno de los servicios públicos municipales. Además, esta herramienta ofrece una visión comparativa de la situación de cada una de las entidades municipales, al estar fundamentada su construcción en una clasificación previa, basada en el tamaño poblacional de los municipios objeto de estudio y en una delimitación de umbrales de cobertura del servicio por tamaño de hábitat (Pereira López, 2013) (Lagares Diez, Pereira López y Jaráiz Gulías, 2015).

Esta metodología que está dando forma a la construcción de un Cuadro de Mando Integral de los servicios públicos a nivel municipal, no se trata de un sistema de indicadores tradicionales sino, tal y como lo entendían Kaplan y Norton (1996) (Kaplan y Norton, 2001a) (Kaplan y Norton, 2001b), un instrumento más complejo, pues combina indicadores y datos de percepción para la elaboración de herramientas, en este caso índices, que facilitan la toma de decisiones en el ámbito de gestión de los servicios públicos.

En este sentido, el cuadro de mando que se propone, tiene su primera expresión en la recopilación de información sobre indicadores objetivos que dan cuenta de los diferentes elementos internos o ítems del servicio. En concreto se recogen un total de 487 indicadores, una media de 27 por cada uno de los dieciocho servicios ${ }^{10}$ que son analizados en el proyecto (ver tabla 1). Dichos indicadores proceden de fuentes públicas y no publicadas; en este último caso, tras un intenso trabajo de campo llevado a cabo con los técnicos de gestión de los diferentes ayuntamientos, en un claro intento de implicar a la organización - las administraciones municipales - en la evaluación y gestión de los servicios que prestan ${ }^{11}$.

9 Si bien los índices de satisfacción proliferaron notablemente desde los años ochenta y noventa (Customer Satisfaction Index (CSI), Swedish Customer Satisfaction Barometer (SCSB),...), como herramientas de análisis de la satisfacción con los servicios, sean éstos de prestación pública o privada (Fornell, 1992); en los últimos años han aparecido nuevas experiencias que abogan por otro tipo de metodologías de carácter mixto, como la que aquí se presenta, donde se intenta construir catálogos o sistemas de indicadores e índices que permitan una evaluación más completa de los servicios (Batet i Lamaña, 2001).

${ }^{10}$ Los dieciocho servicios o áreas analizadas en el proyecto son: área de abastecimiento y saneamiento, área de actividad y empleo, área de cohesión social, área de energías renovables, área de equipamientos culturales, área de equipamientos deportivos, área de educación, área de infraestructuras viarias, área de limpieza viaria y recogida de residuos, área de nuevas tecnologías de la información, área de parques y jardines, área de participación ciudadana, área de sanidad, área de seguridad ciudadana, área de servicios sociales, área de transporte y movilidad, área de urbanismo y vivienda, área de gestión administrativa, transparencia y documentación. Si bien algunos de ellos no son competencia municipal, entendíamos que dada su importancia para la satisfacción de los ciudadanos con la prestación a nivel municipal, se volvía indispensable incluirlos en el análisis (Pereira López, 2013) (Lagares Diez, Pereira López y Jaráiz Gulías, 2015). Se recogen además, datos básicos del municipio, así como datos relativos a la estructura económica, para completar la radiografía del estado actual del municipio.

${ }^{11}$ Es conveniente resaltar en este punto que la opinión y experiencia de los técnicos de gestión se ha tenido en cuenta en varias fases del proceso, no sólo en la recogida de indicadores; sino también en el diseño del sistema o catálogo de indicadores con la realización de varios grupos de discusión, integrados por técnicos de ayuntamientos de los diferentes grupos poblacionales. 
Por otro lado, la puesta en valor de la percepción de los ciudadanos sobre los diferentes elementos que conforman los servicios públicos, así como la satisfacción que sobre los mismos expresan; hacen de esta metodología una magnífica herramienta para la mejora de la gestión que de los servicios públicos llevan a cabo las entidades públicas, concretamente los municipios. La inclusión de la satisfacción como uno de los elementos vertebradores del ICS, facilita la evaluación del servicio y proporciona un replanteamiento en términos de eficiencia y eficacia de la gestión del mismo; al tiempo que pone en valor la importancia del ciudadano en todo el proceso, entendiendo, como señala la literatura ligada al paradigma del Nuevo Servicio Público (Denhardt y Denhardt, 2011) (De Leon y Denhardt, 2000), que los servicios deben estar orientados a los ciudadanos, elemento diferenciador de la prestación en el terreno público respecto al ámbito privado.

Tabla 1. Número de indicadores recogidos por área

\begin{tabular}{|l|c|c|}
\hline \multicolumn{1}{|c|}{ Área o servicio } & $\begin{array}{c}\text { Número de } \\
\text { indicadores } \\
\text { recogidos }\end{array}$ & $\begin{array}{c}\text { Número de } \\
\text { indicadores ICS }\end{array}$ \\
\hline Área de abastecimiento y saneamiento & 23 & 12 \\
\hline Área de actividad y empleo & 21 & 5 \\
\hline Área de cohesión social & 18 & 6 \\
\hline Área de energías renovables & 4 & 3 \\
\hline Área de equipamientos culturales & 12 & 9 \\
\hline Área de equipamientos deportivos & 18 & 6 \\
\hline Área de educación & 40 & 14 \\
\hline Área de infraestructuras viarias & 24 & 8 \\
\hline Área de limpieza y recogida de residuos & 27 & 12 \\
\hline Área de nuevas tecnologías & 7 & 5 \\
\hline Área de parques y jardines & 5 & 5 \\
\hline Área de participación ciudadana & 74 & 9 \\
\hline Área de sanidad & 25 & 10 \\
\hline Área de seguridad ciudadana & 15 & 11 \\
\hline Área de servicios sociales & 18 & 4 \\
\hline Área de transporte y movilidad & 5 & 12 \\
\hline Área de urbanismo y vivienda & 57 & 13 \\
\hline $\begin{array}{l}\text { Área de gestión administrativa, transparencia y } \\
\text { documentación }\end{array}$ & 11 & \\
\hline & & - \\
\hline Datos básicos del municipio & 32 & - \\
\hline Datos de estructura económica del municipio & 51 & \\
\hline
\end{tabular}

Fuente: elaboración propia.

La metodología diseñada pone de esta forma en valor las necesidades y demandas expresadas por los ciudadanos, sus propias percepciones sobre el servicio que reciben 
y del cual son arte y parte. Unos ciudadanos que son sujetos activos de derechos y deberes políticos, dejando a un lado la clásica concepción del ciudadano como cliente y reivindicando por tanto, una noción más rica del concepto de ciudadanía (Denhardt y Denhardt, 2011). En esta secuencia, surge la necesidad de dotar de información al ciudadano para que éste pueda ejercer de forma efectiva los derechos que le son inherentes; por lo que iniciativas metodológicas como la que aquí se presenta, son piezas fundamentales no sólo para dotar de dicha información al ciudadano, sino también para conseguir su implicación efectiva en el proceso de gestión, favoreciendo al mismo tiempo el proceso de rendición de cuentas.

El cuadro de mando se ha diseñado en varias etapas y por ello, también se pueden vislumbrar en él, varios niveles en base al tipo de indicadores e índices de gestión que contiene. Estamos hablando de un amplio conjunto de indicadores e índices de inputs, ouputs y de resultados, que proporcionan no sólo tener una primera aproximación a la situación de cada municipio en lo que a la prestación o cobertura de los servicios públicos se refiere, sino que facilitan, entre otras cuestiones, la identificación de los puntos débiles y/o fuertes de cada uno de los servicios, así como la determinación de los niveles de prestación o cobertura en términos comparados (ver esquema 1).

\section{Esquema 1. Niveles del Cuadro de Mando}

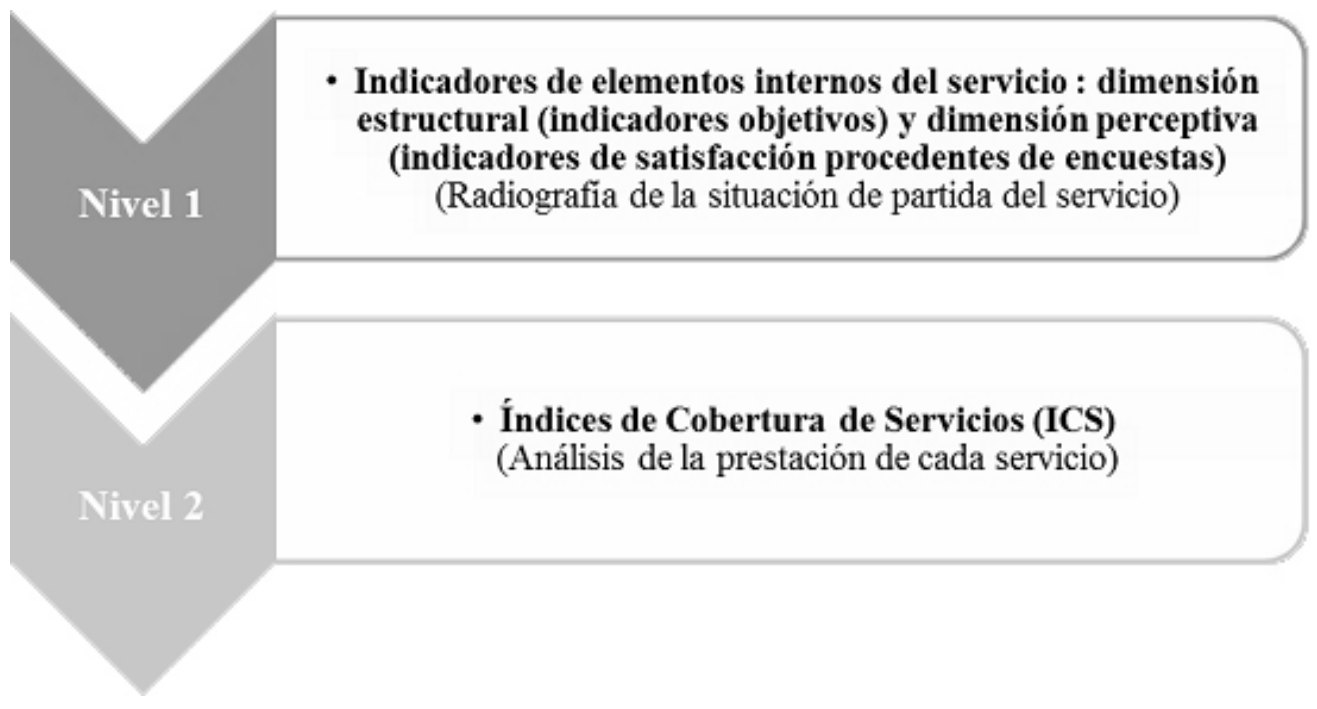

Fuente: elaboración propia.

En un primer nivel de análisis contamos con los valores de los indicadores que han sido elegidos para medir los diferentes elementos internos o items del servicio. Si bien en la mayor parte de los casos se produce univocidad en la correspondencia entre indi- 
cadores y elementos del servicio, en otras ocasiones son más de uno los indicadores que permiten medir y definir el elemento interno del servicio. El establecimiento de esta correspondencia no se ha producido al azar, por el contrario, se ha llegado a la misma tras un proceso complejo en el que han confluido la experiencia aportada por los técnicos de gestión de los municipios, con las percepciones que sobre los diferentes servicios y sus elementos han expresado los ciudadanos de los ayuntamientos. Todo ello ha sido ensamblado mediante un profundo análisis estadístico multivariante, que ha permitido no sólo establecer dicha correspondencia, sino también delimitar el peso que los datos objetivos y los datos perceptivos tienen sobre la configuración final del nivel de satisfacción con cada uno de los servicios públicos analizados ${ }^{12}$.

La primera aportación de los técnicos de gestión de los diferentes ayuntamientos vino dada por los resultados obtenidos en los grupos de discusión llevados a cabo, los cuales ofrecieron información detallada sobre la prestación que de los diferentes servicios llevan a cabo los municipios, así como también datos sobre las debilidades y potencialidades en cada uno de ellos. Las conclusiones de estos grupos fueron determinantes a la hora de identificar cuáles eran los elementos definitorios de los diferentes servicios sobre los que era necesario establecer indicadores, así como las preguntas que era necesario plantear en los estudios cuantitativos que se realizaron con posterioridad. De esta forma, la metodología presentada implicó al mismo tiempo a los miembros de la organización y a los beneficiarios de los servicios que ésta prestaba, en la elaboración de una futura herramienta de evaluación de la gestión y cobertura que de los diferentes servicios se lleva a cabo.

Una vez definidos los indicadores objetivos que conformarían el primer análisis del servicio, ofreciendo una visión descriptiva de la situación de cada uno en los diferentes municipios; y los estudios demoscópicos que permiten medir la satisfacción y percepción de los ciudadanos con dichos servicios, se perfiló un segundo nivel en la elaboración de este cuadro de mando. Un segundo nivel en el que se fusionaron ambos elementos mediante la construcción de un índice sintético que permitiera poner en conexión dos dimensiones fundamentales; por un lado, una dimensión estructural del servicio, la aportada por los indicadores objetivos recabados y que refleja la visión del servicio en la que se ve implicada la organización y por otro lado $^{13}$, la dimensión perceptiva, delimitada por el análisis de las percepciones que sobre los diferentes servicios y sus elementos internos tienen los ciudadanos, usuarios directos o indirectos de dichos servicios. De esta forma, se superó la visión de los tradicionales índices de satisfacción del servicio y se avanzó hacia una concepción innovadora de las herramientas de evaluación y gestión, que culminó con el diseño del Índice de Cobertura de Servicios.

12 Para más información sobre la fundamentación estadística del modelo, ver Pereira López (2013).

${ }^{13}$ Como se desprende de lo recogido en la tabla 1 , no todos los indicadores recogidos han sido empleados a la hora de calcular el valor del ICS para cada una de las áreas, sólo aquellos que en base a los análisis cualitativos y cuantitativos previos se mostraron como determinantes para el análisis de la prestación del servicio en relación a la satisfacción ciudadana expresada con el mismo. 
Tabla 2. Ejemplo de funcionamiento de la metodología en un municipio tipo en cuatro áreas o servicios

\begin{tabular}{|c|c|c|c|c|c|}
\hline Servicio & Ítems del servicio & $\begin{array}{c}\text { Pesos } \\
\text { elementos } \\
\text { internos del } \\
\text { servicio }\end{array}$ & $\begin{array}{c}\text { Valor } \\
\text { elementos } \\
\text { internos del } \\
\text { servicio }\end{array}$ & Valor ICS & $\begin{array}{c}\text { Umbrales } \\
\text { de } \\
\text { cobertura }\end{array}$ \\
\hline \multirow{6}{*}{$\begin{array}{l}\text { Equipamientos } \\
\text { culturales }\end{array}$} & Programas culturales & 0,186 & $-0,010$ & \multirow{6}{*}{0,608} & \multirow{6}{*}{$\begin{array}{c}\text { U. Inferior: } \\
\quad 0,388 \\
\text { U.Superior: } \\
0,586\end{array}$} \\
\hline & $\begin{array}{l}\text { Calidad Instalaciones e infraestructuras } \\
\text { culturales }\end{array}$ & 0,161 & 0,120 & & \\
\hline & Información actividades culturales & 0,171 & 0,001 & & \\
\hline & Dotación equipamientos culturales & 0,188 & 0,041 & & \\
\hline & Centros cívicos & 0,166 & 0,037 & & \\
\hline & Bibliotecas & 0,129 & 0,028 & & \\
\hline \multirow{8}{*}{ Educación } & Programas de conciliación laboral & 0,132 & $-0,029$ & \multirow{8}{*}{0,346} & \multirow{8}{*}{$\begin{array}{c}\text { U. Inferior: } \\
\quad 0,385 \\
\text { U.Superior: } \\
\quad 0,685\end{array}$} \\
\hline & Comedores escolares & 0,137 & 0,002 & & \\
\hline & Transporte escolar & 0,121 & 0,018 & & \\
\hline & Centros escolares & 0,124 & $-0,038$ & & \\
\hline & Profesorado centros educativos & 0,128 & $-0,035$ & & \\
\hline & Dotación centros educativos & 0,119 & $-0,148$ & & \\
\hline & Guarderías públicas & 0,108 & $-0,001$ & & \\
\hline & Educación infantil & 0,131 & $-0,077$ & & \\
\hline \multirow{5}{*}{$\begin{array}{l}\text { Limpieza viaria } \\
\text { y recogida de } \\
\text { residuos }\end{array}$} & Frecuencia servicio de recogida de residuos & 0,181 & $-0,325$ & \multirow{5}{*}{0,391} & \multirow{5}{*}{$\begin{array}{c}\text { U. Inferior: } \\
0,403 \\
\text { U.Superior: } \\
0,614\end{array}$} \\
\hline & Frecuencia servicio de limpieza viaria & 0,216 & $-0,041$ & & \\
\hline & Puntos limpios & 0,205 & $-0,004$ & & \\
\hline & Recursos humanos del servicio & 0,207 & $-0,082$ & & \\
\hline & Recogida selectiva & 0,190 & 0,227 & & \\
\hline \multirow{4}{*}{$\begin{array}{l}\text { Transporte y } \\
\text { movilidad }\end{array}$} & Transporte urbano e interurbano & 0,295 & 0,063 & \multirow{4}{*}{0,497} & \multirow{2}{*}{$\begin{array}{c}\text { U. Inferior: } \\
0,451\end{array}$} \\
\hline & Paradas & 0,293 & 0,004 & & \\
\hline & Taxis & 0,196 & $-0,006$ & & \multirow{2}{*}{$\begin{array}{l}\text { U.Superior: } \\
0,594\end{array}$} \\
\hline & Transporte público adaptado & 0,217 & $-0,067$ & & \\
\hline
\end{tabular}

Fuente: elaboración propia.

En la tabla anterior se presentan a modo de ejemplo, los valores tanto de los indicadores como del ICS para cuatro servicios de un ayuntamiento tipo, concretamente: equipamientos culturales, educación, limpieza viaria y recogida de residuos y servicios sociales. Todas las cuestiones que entorno a la metodología han sido expuestas en líneas anteriores, pueden ser observadas claramente en dicha tabla, de tal forma que el lector puede observar la dimensión perceptiva (pesos de los elementos internos del servicio), el valor de cada uno de los elementos internos del servicio, el valor del índice para ese municipio en esa área concreta y finalmente, los umbrales inferiores y superiores de cobertura entre los que oscila el valor del ICS para los ayuntamientos de la franja poblacional a la que pertenece el municipio escogido (menos de 20.001-50.000 habitantes en este caso) y que permiten interpretar el valor del índice obtenido. 
Queda patente por tanto, cómo el valor del Índice de Cobertura de Servicios alcanzado por un municipio en una determinada área no sólo puede ser interpretado en relación a los municipios del tamaño poblacional al que pertenece, en el caso del servicio de limpieza viaria y recogida de residuos, ligeramente inferior al umbral inferior establecido; sino que también se pueden determinar, cuáles son los elementos del servicio que ejercen una mayor o menor influencia en dicho valor y a qué es debido, así pues, en el caso del área de educación observamos como el elemento relativo a la dotación de los centros educativos ejerce una tracción negativa sobre el valor final del ICS, lo que nos permitiría afirmar que se trata de un elemento «crítico» sobre el que se debe poner hincapié en términos de gestión, puesto que los indicadores objetivos que nos han permitido construir su valor, presentan un déficit ${ }^{14}$. Y aún más, la dimensión perceptiva expresada en la tercera columna de la tabla 2 , nos da una información de gran interés, los pesos de los elementos internos del servicio ${ }^{15}$, es decir, la priorización que en términos de satisfacción realiza la ciudadanía; y que permite determinar si la cobertura o prestación realizada de un servicio se encuentra o no saturada en términos de satisfacción con el mismo.

Respecto a la detección de los denominados como elementos «críticos», podemos observar en la tabla 2, como en casi todas las áreas existe algún elemento interno del servicio que ejerce una influencia negativa sobre el valor final del índice, lo cual es debido a unos valores deficitarios de los indicadores que permiten la construcción del valor final del elemento. Si bien y bajo la lógica de progreso que guía esta construcción, esta situación no debe ser entendida en términos negativos, puesto que permite la detección y por tanto la paliación de la misma en términos de gestión.

Así mismo, la tabla nos permite comprobar claramente cuáles son los tres posibles comportamientos que pueden producirse en la aplicación del ICS. Un valor del índice por encima del umbral superior establecido, como es el caso del servicio de cultura, lo cual sería indicativo de que el servicio prestado en este municipio presenta una cobertura notable y satisfactoria; un valor del ICS dentro de los umbrales establecidos, como es el caso del servicio de transporte y movilidad, por lo que se podría decir que el servicio prestado en este caso presenta una cobertura adecuada; y finalmente dos valores por debajo de los umbrales inferiores establecidos, en las áreas de limpieza y educación, que nos permiten afirmar que la cobertura no es adecuada y por tanto resulta insatisfactoria.

14 En este caso concreto, los indicadores que reflejan unos valores que llevan a que la construcción de este elemento interno del servicio, dotación centros educativos, traccione negativamente son: existencia y número de salas de informática, existencia y número de aulas de docencia con ordenadores, existencia y número de bibliotecas y existencia y número de instalaciones deportivas; todos ellos para el conjunto de los centros educativos del municipio.

${ }^{15}$ Estos pesos son el resultado de la aplicación de análisis multivariante a los resultados de los estudios demoscópicos realizados. Para más información ver Pereira López (2013) y Lagares Diez, Pereira López y Jaráiz Gulías (2015). Además son valores comunes para cada uno de los tamaños de hábitat, teniendo en cuenta la lógica comparativa establecida y el hecho de que se asume que diferentes tamaños de hábitat implican también diferencias en términos de satisfacción con los servicios. 
En definitiva, el ICS es un único valor por servicio y municipio, que permite conectar dos fuentes de información, simplificando la lectura que sobre la cobertura del servicio se lleva a cabo, en relación a unos umbrales que permiten su interpretación y que están definidos en base a los grupos de municipios determinados previamente. Pero más allá del valor del índice y de su interpretación, su propia construcción, basada en los elementos objetivos y perceptivos, permite determinar no sólo el nivel de cobertura general de un servicio en relación a los niveles de cobertura de los municipios de similares características; sino delimitar cuáles son los elementos del servicio que ejercen una mayor o menor influencia en dicho nivel, estableciendo por tanto, lo que podríamos denominar como elementos «críticos» del servicio (ver tabla 2). Y ello es posible en gran parte, a la concepción integral que ha guiado y guía la construcción de esta metodología, un cuadro de mando global de análisis y evaluación de la cobertura de los servicios públicos de ámbito municipal.

\section{Nuevos avances en el diseño de la metodología}

En la actualidad, el Equipo de Investigaciones Políticas que ha llevado a cabo este proyecto y cuya aplicación se sigue llevando a cabo en los municipios de la provincia de A Coruña, continúa trabajando y avanzando en su desarrollo, con el diseño y testeo de índices de rendimiento económico. Unos índices con los cuáles se sumará al análisis aportado por el ICS, la importancia de la optimización de los recursos económicos destinados a la cobertura o prestación de los servicios públicos, por parte de las entidades públicas.

Estos índices han sido concebidos como indicadores económicos de impacto que posibilitarán a los técnicos y gestores (en el caso concreto que nos ocupa, la Diputación de A Coruña) basándose en sus resultados, proceder a una eficiente y eficaz redistribución de los recursos económicos en dos sentidos: en el volumen de recursos destinados en general a los diferentes servicios públicos y por otro lado, al volumen de recursos y número de proyectos puestos en marcha en cada uno de los municipios a los que representa.

Una distribución en la cual se podrá intentar priorizar entre aquellos servicios que, en cada uno de los municipios, presentan déficits de cobertura en términos relativos a los municipios de su mismo tamaño poblacional; teniendo en cuenta la incidencia de las inversiones económicas en las variaciones de calidad del servicio, en base a los parámetros objetivos y a las percepciones y niveles de satisfacción de los ciudadanos con los mismos. El propio diseño de estos índices supone dar un paso más en la gestión estratégica de los recursos, de las inversiones y de los fondos que la entidad provincial pone a disposición de los municipios; conectando la información extraída de los índices de cobertura con el rendimiento de las actuaciones de la Diputación.

De esta forma, se podrá conocer si las inversiones realizadas inciden sobre los elementos críticos internos de cada servicio que tienen más impacto en los niveles de satisfacción ciudadana, y así, tener la posibilidad de evaluar y, de ser el caso, reorientar, las mencionadas inversiones. En otras palabras, el hecho de que tengamos identificados aquellos elementos internos de cada servicio con mayor impacto en la satis- 
facción, revela un importante conocimiento a la hora de analizar si las inversiones realizadas influyen de algún modo en el índice de cobertura de dichos servicios. En consecuencia, el estudio del rendimiento de las inversiones realizadas, aportará luz para el diseño de una planificación estratégica centrada en una redistribución más eficaz y eficiente de los recursos, permitiendo a la administración reequilibrar la balanza de su gestión (Pereira López, 2013) (Lagares Diez, Pereira López y Jaráiz Gulías, 2015).

\section{Conclusiones}

La metodología diseñada, de la cual el Índice de Cobertura de Servicios es pieza fundamental, se revela como un instrumento idóneo para la evaluación de la cobertura de los servicios públicos municipales y la reorientación de la gestión de los mismos por parte de las administraciones y gestores públicos:

- El hecho de que base su construcción en unidades de parámetros similares (tamaños de hábitat) facilita notablemente la realización de comparaciones, no sólo entre unidades de análisis, sino también entre servicios.

- La combinación en el diseño del cuadro de mando de técnicas cuantitativas y cualitativas ha permitido delimitar un número de indicadores óptimo para cada uno de los servicios, buscando simplicidad pero sin renunciar a captar los diferentes aspectos o ítems de cada uno de ellos.

- La fusión de elementos estructurales y perceptivos proporciona una visión más compleja y profunda de cada servicio, evitando caer en el error de pensar que un único indicador pueda dar cuenta de la situación general de un servicio.

- El uso de diversas técnicas, así como el análisis y fundamentación estadística de los modelos, ha permitido contrastar la idoneidad de los indicadores utilizados en la medición de cada servicio.

- Además, su diseño y fácil retroalimentación, potencian su perdurabilidad en el tiempo, garantizando la mejora continua del servicio, lo que la convierte por tanto, en un mecanismo de mejora y progreso para la gestión que de los servicios públicos llevan a cabo las entidades locales.

- Por último y como valor máximo de esta metodología, la implicación de los ciudadanos en el proceso de gestión, a través de la realización de estudios de satisfacción con los servicios públicos, garantiza que los objetivos establecidos en la gestión del servicio se adecúen a sus demandas y necesidades.

Los resultados que han sido extraídos de las dos primeras aplicaciones del ICS (2013 y 2014) han arrojado datos de gran interés sobre los niveles de cobertura de los diferentes servicios en términos generales y en relación a los diferentes tamaños poblacionales. Así pues, se ha podido determinar cómo existen áreas en las que se generan mayores disparidades en cuanto a los niveles de cobertura entre los distintos grupos poblacionales, tal y como puede ser el caso del servicio de limpieza viaria y recogida de residuos; y otros servicios, en los cuales esas diferencias son apenas apreciables, véase el caso del área de gestión administrativa, transparencia y documentación. Al mismo tiempo, se ha podido determinar la existencia de elementos internos del servi- 
cio que se han desvelado como «críticos» dentro del mismo para cada uno de los ayuntamientos, habitualmente por la escasa cobertura del/os mismo/s. Por otro lado, también se ha podido establecer la existencia de algunos elementos internos que podríamos denominar de «situación especial», en los cuales, la dificultad de conseguir de forma sistemática dicha información, genera ciertos problemas de cobertura para el conjunto general de los municipios, independientemente del tamaño poblacional, es el caso de los ítems relativos al área de seguridad ciudadana.

Si bien son muchos los retos que quedan por delante en el diseño final y contrastación del funcionamiento de este Cuadro de Mando Integral; las primeras aplicaciones llevadas a cabo permiten avanzar la robustez de esta herramienta de análisis, así como su gran utilidad en la gestión que de los servicios públicos llevan a cabo las administraciones públicas.

\section{Bibliografía}

(12 de 12 de 2001). Ley 18/2001 General de Estabilidad Presupuestaria. Madrid, España.

(26 de Noviembre de 2003). Ley 47/2003 General Presupuestaria. Madrid, España.

(16 de 12 de 2003). Ley 57/2003 de medidas para la modernización del gobierno local. Madrid, España.

(23 de 11 de 2004). Orden EHA/4041/2004 de Instrucción del modelo normal de contabilidad local. Madrid, España.

Amo Baraybar, F. (2011). El Cuadro de Mando Integral «Balanced scorecard». ESIC Editorial, Madrid.

Asociación Española de Contabilidad y Administración de Empresas, A. (1999). Un sistema de indicadores de gestión para las entidades públicas. Documents, 16.

Audit Commission, A. (2000). On target. The practice of performance indicators. Audit Commission, London.

Bastidas E. y Ripoll, V. (2003). «Una aproximación a las implicaciones del cuadro de mando integral en las organizaciones del sector público», en Comendium, pp. 23-41.

Batet i Lamaña, M.; Pastos i Millan, J.R.; Carreras i Puigdengol, J.M. y Mora i Puigví, A. (2001). Propuesta de indicadores básicos de gestión de servicios públicos locales. Fundació Carles Pi i Sunyer d'Estudis Autonómics i Locals, Barcelona.

Batet Lamaña, M. y Mora Puigví, A. (2001). «Indicadores de gestión de servicios públicos locales: una iniciativa desde Cataluña» en V. S. Local, Evaluación y control de políticas públicas. Gijón: Ayuntamiento de Gijón.

Brignall, S. y Modell, S. (2000). «An institucional perspective on performance measurement and management in the new sector public», en Management Accounting Research, $\mathrm{n}^{\circ} 11$, pp. 281-306.

Brusca Alijarde, M. (1997). «Una nueva perspectiva de los indicadores financieros en contabilidad pública: los indicadores de gestión», en Revista de Contabilidad, nº 0, vol. 0, pp. 2756.

Colino, C. y Del Pino, E. (2008). «Democracia participativa en el nivel local; debates y experiencias en Europa», en Revista Catalana de Dret Públic, nº 37, pp. 247-283.

De Pablos Escobar, L. y Valiño Castro, A. (2000). Economía del gasto público: control y evaluación. Civitas, Madrid. 
de Leon, L. y Denhardt. (2000). «The political theory of reinvention», en Public Administration Review, vol. 2, nº 60, pp 89-97.

Denhardt, R.B. y Denhardt, J. V. (1999). Leadership for change: case studies in American local government. Arlington: Pricewaterhouse Coopers Endowment for the Business of Government.

Denhardt, R.B. y Denhardt, J.V. (2011). «The new public service: serving rather than steering», en Public Administration Review, vol. 6, n 60, pp. 549-559.

Font, J, y Gomá, R. (1999). «La participación en la política local» en Fundación Encuentro (ed.) Informe España 2000. Fundación Encuentro, Madrid.

Fornell, C. (1992). «A National Customer Satisfaction Barometer: the Swedish experience», en Journal of Marketing, vol. 1, $\mathrm{n}^{\circ}$ 56, pp. 6-21.

Governmental Accounting Standards Board, G. (1987). Concepts statement No.1. Objectives of financial reporting. GASB, Stamford.

Governmental Accounting Standards Board, G. (1994). Concepts statements No.2, Objectives of financial reporting. GASB, Stamford.

Hoque, Z. (2014). «20 years of studies on the balanced scorecard: tends, accomplishments, gaps and opportunities for future research», en The Bristish Accounting Review, n 46, pp. 33 59.

Kaplan, R. S. (2012). «The balanced scorecard: comments on balanced scorecard commentaries», en Journal of Accounting \& Organizational Change, vol. 4, nº 8, pp. 539-545.

Kaplan, R. y Norton, D. (2006a). «How to implement a new strategy without disrupting your organization», en Harvard Business Review, vol. 84, nº 3, pp. 100-109.

Kaplan, R. y Norton, D. (2006b). Alignment: Using the balanced scorecard to create corporate synergies. Harvard Business School Press, Boston MA.

Kaplan, R. y Norton, D. (2001a). «Transforming the balanced scorecard from performance measurement to strategic management: Part I», en Accounting Horizons, vol. 1, $\mathrm{n}^{\mathrm{o}}$ 15, pp. 87-104.

Kaplan, R. y Norton, D. (2001b). «Transforming the balanced scorecard from performance measurement to strategic management: Part II», en Accounting Horizons, vol. 2, $\mathrm{n}^{\circ}$ 15, pp. 147-160.

Kaplan, R. S. (2000). El cuadro de mando integral: the balanced scorecard. Gestión 2000, D.L, Barcelona.

Keehley, P.; Medlin, S. y MacBride, S. (1997). Benchmarking for best practices in the public sector. CA: Jossey-Bas, Inc, San Francisco.

Kaplan, R. y Norton, D. (1996). «Using the balanced scorecard as strategic management system», en Harvard Business Review, pp. 75-85.

Kloot, L. y Martin, J. (2000). «Strategic performance management: a balance approach to performance management issues in local government», en Management Accounting Research, $n^{\circ} 11$, pp. 231-251.

Lagares Diez, N., Pereira López, M. y Jaráiz Gulías, E. (2015). «El Índice de Cobertura de Servicios (ICS). Un instrumento para la evaluación de los servicios públicos», en Gestión y Análisis de Políticas Públicas, $\mathrm{n}^{\circ} 13$ (en prensa).

López Hernández, A. y Ortiz Rodríguez, D. (2004). «Los indicadores de gestión y control de eficiencia del sector público», en Revista Española de Control Externo, vol 6, n 18, pp. 189-220.

López, A.M.; Navarro Galera, A. y Ortiz, D. (2001). «Indicadores de gestión de servicios públicos locales: el proyecto SINIGAL» en V. S. Local, Evaluación y control de políticas públicas. Indicadores de gestión. Ayuntamiento de Gijón. 
Navarro Galera, A.; Ortiz Rodríguez, D. y López Hernández, A.M. (2006). «La aplicación de indicadores de gestión en la administración local: una propuesta para facilitar su viabilidad», en IX Jornadas de Trabajo sobre Contabilidad Pública. Logroño.

Pereira López, M. (Noviembre de 2013). Modelos de construcción de indicadores para la evaluación de los servicios públicos: definición y funcionamiento del Índice de Cobertura de Servicios. Tesis doctoral. Santiago de Compostela.

Sorensen, J.E. y Grove, H.D. (1977). «Cost-outcomes and cost-effectiveness analysis: emerging nonprofit performance evaluation techniques», en The Accounting Review, vol. 3, $\mathrm{n}^{\mathrm{o}}$ 52, pp. 658-675.

Torres Pradas, L. (1991). «Indicadores de gestión para las entidades públicas», en Revista Española de Financiación y Contabilidad, vol. 21, nº 67, pp. 535-558.

Wolf, C. (1995). Mercados o gobiernos. Elegir entre alternativas imperfectas (Vol. 6). Instituto de Estudios Fiscales, Madrid. 